
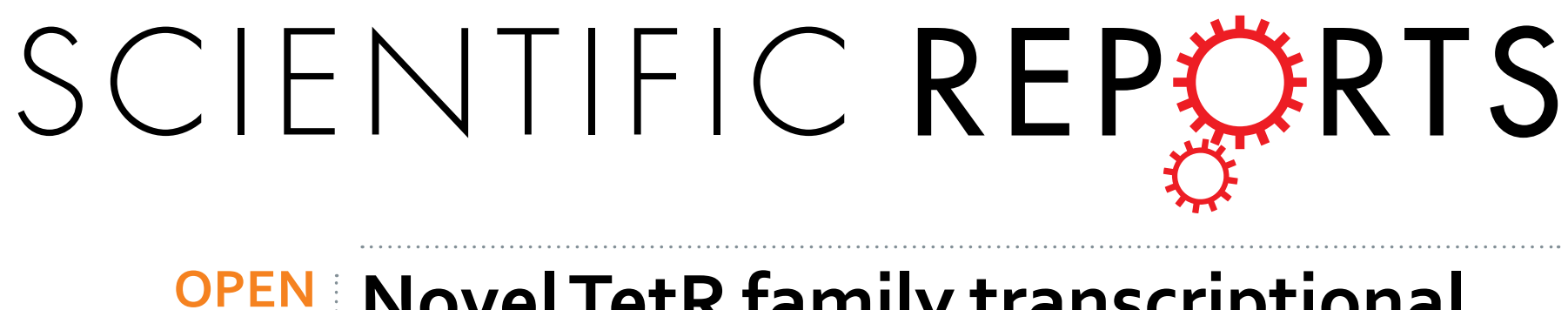

Novel TetR family transcriptional
factor regulates expression of
multiple transport-related genes

Received: 05 April 2016

Accepted: 17 May 2016

and affects rifampicin resistance in

Published: 07 June 2016

\title{
Mycobacterium smegmatis
}

\author{
Huicong Liu, Min Yang \& Zheng-Guo He
}

Transport-related genes significantly affect bacterial antibiotic resistance. However, the effects of these genes and their regulation of bacterial drug resistance in several mycobacterial species, including the fast-growing Mycobacterium smegmatis, the pathogen M. tuberculosis and M. avium have not been clearly characterized. We identified Ms4022 (MSMEG_4022) as a novel TetR family regulator that activates the expression of seven transport-related genes and affects drug resistance in $M$. smegmatis. Overexpression of Ms4022 inhibited $M$. smegmatis growth and enhanced mycobacterial resistance to the anti-tuberculosis drug rifampicin (RIF). By contrast, the Ms4022-deleted mycobacterial strain has shown sensitive to RIF. Ms4022 recognized three 19 bp non-palindromic motifs containing a 9 bp conserved region at their $5^{\prime}$ end and it directly regulated seven transport-related genes, which affects mycobacterial resistance to RIF. Overexpression of three of seven transport-related genes (Ms1448, Ms1613, and Ms5278) inhibited the growth of $M$. smegmatis. This study improves our understanding of the function of mycobacterial transport-related genes and their regulation of bacterial drug resistance.

The occurrence and spread of bacterial drug resistance, especially multi-drug resistance (MDR), in several important human pathogens (e.g., Mycobacterium tuberculosis) pose a serious threat to human health worldwide. Recent studies have indicated that the existence of drug efflux systems, which can pump drugs out of bacterial cells and result in bacterial resistance to drugs, is one of the important causes of bacterial antibiotic resistance ${ }^{1,2}$. However, the regulatory mechanisms of bacterial drug resistance involving drug efflux remain unclear.

M. tuberculosis (MTB), the causative agent of tuberculosis (TB), continues to be a major global health prob$\mathrm{lem}^{3}$. Several mycobacterial species have different efflux pump genes associated with resistance to multiple drugs $^{4-6}$. For example, LfrA is a multidrug efflux pump and activates the multidrug efflux in M. smegmatis ${ }^{7}$. The iniBAC operon encodes a typical efflux pump and could function as an MDR pump system in M. smegmatis and $M$. tuberculosis ${ }^{8}$. Multidrug-resistant and extensively drug-resistant $M$. tuberculosis strains frequently exhibit increased expression of this efflux system 9 . Many drug transporter regulatory proteins, including activators and repressors, have been identified in recent years. TetR family transcription factors regulate diverse physiological functions in bacteria. They control physiological processes, such as catabolic pathways, biosynthesis of antibiotics, and osmotic stress, in the pathogenicity of gram-negative and gram-positive bacteria ${ }^{10}$. The members of this family are often employed as negative regulators that inhibit the expression of target genes. For example, EmrR in Escherichia coli and QacR in Staphylococcus aureus negatively regulate MDR pumps ${ }^{11,12}$. EthR is a repressor of the TetR/CamE family associated with ethionamide resistance in $M$. tuberculosis ${ }^{13}$.

$M$. smegmatis, a fast-growing nonpathogenic mycobacterium, has been widely utilized as a model organism for the study of the mechanisms of gene regulation in extremely slow-growing mycobacterial species, such as M. tuberculosis ${ }^{14,15}$. Interestingly, M. smegmatis is also a suitable model for the study of the regulatory mechanism of mycobacterial drug resistance ${ }^{16}$. In particular, more than 500 potential regulatory factors and 600

National Key Laboratory of Agricultural Microbiology, College of Life Science and Technology, Huazhong Agricultural University, Wuhan 430070, China. Correspondence and requests for materials should be addressed to M.Y. (email: yangmin@mail.hzau.edu.cn) or Z.-G.H. (email: hezhengguo@mail.hzau.edu.cn) 
transport-related genes are encoded by the genome of M. smegmatis (GenBank accession number CP000480). However, the physiological roles of these regulators and transport-related genes and their relationships with bacterial drug resistance remain unknown.

In this study, we characterize a new TetR family transcriptional factor, Ms4022, as a positive regulator in M. smegmatis. Ms4022 activates the expression of seven transport-related genes and affects the RIF resistance and growth of $M$. smegmatis. This study enhances our understanding of transport-related gene regulation and drug resistance in mycobacteria.

\section{Materials and Methods}

Strains, enzymes, plasmid, regents and DNA primers. E.coli $\mathrm{DH} 5 \alpha$ cells were used to construct the recombinant plasmids. E.coli BL21 cells (DE3) and pET28a purchased from Stratagene (La Jolla, CA, USA) were used to express M. smegmatis protein. Restriction enzymes, T4 ligase, dNTPs and all antibiotics were purchased from TaKaRa Biotech (Shiga, Japan). All primers were synthesized by Tsingke Biological Technology (Wuhan, China) (Supplementary Table 1 and 2). DNA purification kits were purchased from Waston Biotechnologies (Wuhan China). All plasmids used in this study were listed in Supplementary Table 3. Antisera were purchased from the Laboratory Animal Centre, Institute of Virology, Chinese Academy of Sciences, Wuhan, China.

The screening of rifampicin (RIF) related transcriptional regulators. Over 500 transcriptional regulator genes were amplified from $M$. smegmatis genomic DNA. The gene fragments were mixed as a pool and cloned into pMV261 vector to construct the regulatory genes overexpression plasmids library. The plasmids library were electrophoretic transferred into M. smegmatis $\mathrm{mc}^{2} 155$, and the strains were screened on $7 \mathrm{H} 10$ plates containing $1.5 \mu \mathrm{g} / \mathrm{mL}$ RIF. As a result, those having increased RIF resistance or decreased RIF susceptibilities were identified as primary candidates. To avoid random mutations that may contribute to RIF resistance, plasmid were extracted from each of the primary candidates, and transformed into the wild type M. smegmatis and assayed thrice in a similar way. In final, the increased RIF resistance is sufficient to attribute to the overexpression of the corresponding transcriptional regulator.

Electrophoretic mobility shift assay. Electrophoretic mobility shift assay (EMSA) was used to detect the DNA binding ability of Ms4022. DNA fragments for the DNA binding activity assays were from M. smegmatis genomic DNA or synthesized directly by Tsingke Biological Technology (Wuhan, China). The reaction $(20 \mu \mathrm{l})$

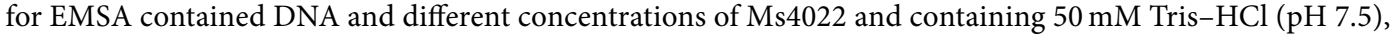
$10 \mathrm{mM} \mathrm{MgCl}_{2}$, and $50 \mathrm{mM} \mathrm{NaCl}$. The DNA and reaction mixtures were incubated at $4{ }^{\circ} \mathrm{C}$ for $30 \mathrm{~min}$ with various amounts of Ms4022, then subjected to 5\% native PAGE using $0.5 \times$ Tris/borate/EDTA (TBE) as running buffer. Electrophoresis was performed at $150 \mathrm{~V}$ at room temperature for $2 \mathrm{hrs}$. Images were acquired by Typhoon Scanner (GE Healthcare, Little Chalfont, Buckinghamshire, UK).

Footprinting assay. The Ms4022 promoter DNA (upstream $500 \mathrm{bp}$ of $M s 4022$ ) was amplified with reverse primer labeled with Fluorescein Isothiocyanate (FITC). The purified DNA fragment was added to the reaction mixture (containing various amounts of Ms4022) at $4^{\circ} \mathrm{C}$ for $30 \mathrm{~min}$ as in EMSA. All of the mixtures were treated with DNaseI (1 unit, Fermentas) (Fermentas China Co., Ltd, Shenzhen China) at $37^{\circ} \mathrm{C}$ for $2 \mathrm{~min} 30 \mathrm{~s}$ as described previously ${ }^{14}$. The results were analyzed with Applied Biosystems 37030XL DNA analyzer (manufactured by Tsingke Company, Wuhan, China).

Chromatin immunoprecipitation Assay. Chromatin immunoprecipitation (ChIP) assay was used to detect the DNA binding ability of Ms4022 in vivo. Ms/wt and Ms/ $\Delta \mathrm{Ms} 4022$ strains were cultivated in $100 \mathrm{ml} 7 \mathrm{H} 9$ medium to $\log$ phase $\left(\mathrm{OD}_{600} \approx 1.0\right) .1 \%$ formaldehyde was used to fix the cells for $30 \mathrm{~min}$, then added $125 \mathrm{mM}$ glycine for $5 \mathrm{~min}$ to stop the reaction, and then the cells were harvest. Cells were treated as described previously ${ }^{15}$. Samples of those were incubated with antibodies of $\mathrm{Ms} 4022$ or preimmune serum for $3 \mathrm{~h}$ at $4{ }^{\circ} \mathrm{C}$. The complexes were treated as described previously ${ }^{15}$. The protocol of PCR amplification contained one denaturation step of $5 \mathrm{~min}$ at $95^{\circ} \mathrm{C}$, then 26 cycles of $1 \mathrm{~min}$ at $95^{\circ} \mathrm{C}, 1 \mathrm{~min}$ at $60^{\circ} \mathrm{C}$ and $1 \mathrm{~min}$ at $72^{\circ} \mathrm{C}$.All of the primers used here were list in supplementary Table 1.

Quantitative real-time PCR. The M. smegmatis wild type strain (Ms/wt) and recombination strains (Ms/ $\Delta$ Ms4022, Ms/pMV261 and Ms/pMV261-Ms4022) were incubated in 7H9 medium to log phase $\left(\mathrm{OD}_{600} \approx 1.0\right)$. Then cells were harvest and storage at $-80^{\circ} \mathrm{C}$. RNA was extracted from these cells as described previously ${ }^{15}$, then digested with DNaseI and was used as template for synthesis of cDNA. Reactions were carried out in a Bio Rad CFX RT-PCR machine (Bio Rad, California, USA). Fold change in gene expression was calculated by the $2^{-\Delta \Delta C t}$ $\operatorname{method}^{17}$.

Determination of Mycobacterium growth curves and the MIC of drugs. All of the recombinant strains grown at $37^{\circ} \mathrm{C}$ in $7 \mathrm{H} 9$ with or without kana to $\log$ phase, then diluted in $100 \mathrm{ml} 7 \mathrm{H} 9$ with or without drugs to an $\mathrm{OD}_{600}$ of 0.1 . Drug concentration is $8 \mu \mathrm{g} / \mathrm{ml}$ Isoniazid (INH), $1.5 \mu \mathrm{g} / \mathrm{ml}$ Rifampicin (RIF) or $1 \mu \mathrm{g} / \mathrm{ml}$ ethambutol (EMB) respectively. All of the strains were grown at $37^{\circ} \mathrm{C}$ at $160 \mathrm{rpm}$ for $3-4 \mathrm{~d}$. These samples were taken by equal time and measured the $\mathrm{OD}_{600}$ as described previously ${ }^{16}$.

For minimal inhibitory concentration (MIC) determination using tube dilution methods as described previously ${ }^{18}$. Mid-log phase strains were diluted up to $0.1 \mathrm{ml}$ culture containing $10^{5} \mathrm{CFUs}$ (colony forming unit) and RIF was two-fold diluted at a volume of $2.5 \mathrm{ml}$ in $7 \mathrm{H} 9$ without Tween-80. All of the strains were grown at $37^{\circ} \mathrm{C}$ at $160 \mathrm{rpm}$ for 3-4d. The minimum concentration that prevented pellet formation was identified as MIC. 
Analysis of $\beta$-galactosidase activity. Promoter - LacZ fusions plasmids were constructed base on pMV261 for analyzing the $\beta$-galactosidase activity experiments as described previously ${ }^{19}$. The multiple clone site of the plasmid was reformed by inserting a MCM stream and the same sense mutation was performed in the $E c o R I$ site of $l a c Z$. Then a pMV261-MCM-LacZ vector was constructed firstly. Promoter of Ms4022 target genes were cloned by EcoRI/XbaI into pMV261-MCM-LacZ (digested with EcoRI/XbaI). The reporter plasmids were electrophorated into Ms/wt and Ms/ $\Delta \mathrm{Ms} 4022$ strains. All recombination strains were grown in $7 \mathrm{H} 9$ at $37^{\circ} \mathrm{C}$ to an OD600 of 0.6 0.8. $\beta$-galactosidase measurements were performed as described previously ${ }^{19}$.

Southern blotting. A pMind derived suicide plasmid containing the hygromycin resistance gene was used to construct Ms 4022 knockout vector. The vector was electrophorated into Ms/wt stain. The Ms/ $\Delta \mathrm{Ms} 4022$ was selected on 7H10 (BD, USA) plate containing $100 \mu \mathrm{g} / \mathrm{ml}$ hygromycin. Ms4022 deleted strain was identified by southern bolt analysis. Ms/wt and Ms/ $\Delta$ Ms4022 genomic DNA were digested with ApaI. The 391bp DNA probe of the upstream of the Ms4022 was amplified using special primers (Supplementary Table 1).

\section{Results}

Ms4022 inhibits cell growth and positively regulates RIF resistance in $\mathbf{M}$. smegmatis. Utilizing the transcriptional factor overexpression library of $M$. smegmatis constructed previously ${ }^{20}$, we screened the regulator-overexpressing strains on $7 \mathrm{H} 10$ plates containing $1.5 \mu \mathrm{g} / \mathrm{mL}$ RIF and identified a TetR family regulator in M. smegmatis, Ms4022 (Fig. 1A), which is associated with RIF resistance. As shown in Fig. 1B, the Ms4022-overexpressing strain (Ms/pMV261-Ms4022) grew slower than the control strain (Fig. 1B, left panel), and Ms/pMV261-Ms4022 showed increased resistance to RIF (Fig. 1B, right panel). Notably, no obvious effect was observed in the presence of INH or EMB (Supplementary Fig. 1). The Ms4022 deleted strain $(\Delta \mathrm{Ms} 4022 /$ pMindD) was more sensitive to RIF (Fig. 1C, right panel), while bacterial growth was unchanged when Ms4022 was deleted in M. smegmatis (Fig. 1C, left panel). The sensitivity of $\Delta$ Ms4022/pMindD to RIF partially recovered when Ms4022 was complemented with pMindD plasmid (Fig. 1C, right panel). The MIC of Ms/ $\Delta$ Ms 4022 $(3.13 \mu \mathrm{g} / \mathrm{ml})$ to RIF was two-fold lower than Ms $/ w t(6.25 \mu \mathrm{g} / \mathrm{ml})$, whereas the MIC of Ms/pMV261-Ms4022 was four-fold higher than that of Ms/pMV261 $(25 \mu \mathrm{g} / \mathrm{ml})$. These results suggest that the TetR family regulator, Ms4022, contributes to the RIF resistance of M. smegmatis.

Ms4022 recognizes three binding sites within the upstream sequence of its own operon. EMSA assay was utilized to detect the binding of Ms4022 to its upstream promoter DNA. As shown in Fig. 2A, the DNA/protein complex shifts significantly increased with increasing amounts of Ms4022 proteins (lanes 6 to 8). However, Ms4022 could not specifically bind to the control promoter Ms4121p (Fig. 2A). Furthermore, competition assay indicated that unlabeled Ms4022p, instead of Ms4121p, competitively inhibited the binding of Ms4022 to the labeled Ms4022 promoter DNA substrate (Fig. 2B). These results prove that Ms 4022 specifically binds to Ms4022p.

DNaseI footprinting assay was then employed to identify the binding motif of Ms4022. The non-coding strand of the DNA substrate was labeled with fluorescein isothiocyanate and then utilized as a substrate in the assay. When the mixture of Ms4022 and its promoter was co-incubated with DNaseI, two protected regions of Ms4022 promoter were detected (Fig. 3A). The first region AATCAGCACTGTGTGGATT extended from positions -57 to -39 (relative to the Ms4022 start codon). The second region AATAAGCACAGTGTCGAAA AA AATAAGCACTCCGTCA $\underline{A} \underline{\underline{T}}$ extended from positions -277 to -238 . Two defective palindromic motifs were separated by two nucleotides (AA). All three motifs had the similar sequence $5^{\prime}$-AATCAGCAC $-3^{\prime}$ in protected regions (Fig. 3B). To characterize the DNA binding motif for Ms4022 protein, DNA fragments in the Ms4022 promoter were amplified by PCR and then used as EMSA substrates. Unlike Ms4121p1, Ms4022 could bind to s1, s2, and s3 (Fig. 3B,C). Multiple binding sites were presumed to exist in the Ms4022 promoter. Different truncated DNA fragments within the Ms4022 promoter were amplified (s4 to s7). In the EMSA assay, Ms4022 bound to s4, s5, and s6 but not to s7 (Fig. 3C and Supplementary Fig. 3B). As previously mentioned, at least two motifs existed. One was in s2, and the other one was located near the translation initiation site of Ms4022.

To evaluate the effect of the motif sequence on Ms4022 recognition, we designed DNA substrates with and without motif mutation (Fig. 4A). The results show that when the conserved $9 \mathrm{bp}$ regions were mutated, Ms 4022 lost its binding ability to DNA fragments F2, F3, F5, and F7 (Fig. 4B).

These results indicate that three binding motifs existed in the promoter of Ms4022. The putative binding motifs for Ms4022 contained an imperfect palindromic sequence motif.

Ms4022 binds with 22 promoters of transport-related genes. A total of 315 potential target genes were identified by using these motifs to search the intergenic region of $M$. smegmatis separately. On the basis of the first, second, and third motifs, 134, 132, and 71 potential target genes were identified. Four promoters, namely, MSMEG_3427p, MSMEG_3799p, MSMEG_4022p, and MSMEG_5035p, had their promoter with the three binding motifs (Fig. 5A). Among them, promoters were randomly selected and applied to EMSA assays to confirm their binding with Ms4022. As a result, 19 of 34, 19 of 21, and 7 of 8 selected promoters bound with Ms4022 protein (Supplementary Fig. 4).

We analyzed the classification of the 315 potential genes by using the categories in the Cluster of Orthologous Groups (COG) of proteins. COG includes 31 membrane-associated transporter genes (Fig. 5B). By using the Web Logo tool ${ }^{21}$, a logo assay containing $19 \mathrm{bp}$ non-palindrome sequence AANNAGCACNCCGTCGA NN was characterized, and the last two bases show very low similarity (Supplementary Fig. 5B). Among the 31 promoters of Ms4022 target genes, 22 promoters were bound by Ms4022 in vitro (Supplementary Fig. 4).

ChIP assays were established to detect the binding ability of Ms4022 in vivo for these promoters. As shown in Fig. 6A (see also Supplementary Fig. 5C), a specific Ms4022 antiserum recovered the target promoters by 
A

Ms4022 domain structure

20 aa

64aa

199 aa

HTH_TetR_N
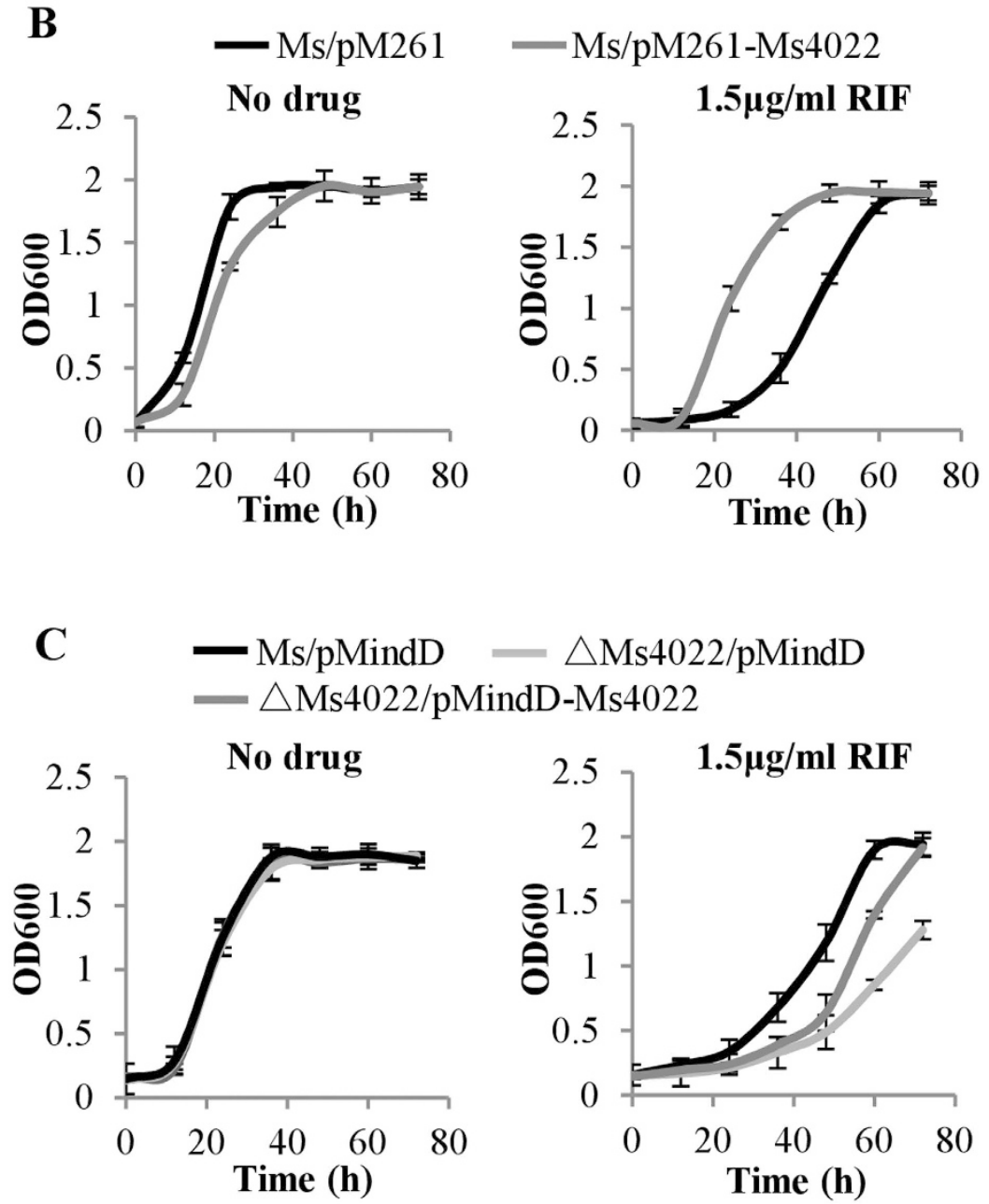

$\triangle \mathrm{Ms} 4022 / \mathrm{pMindD}$

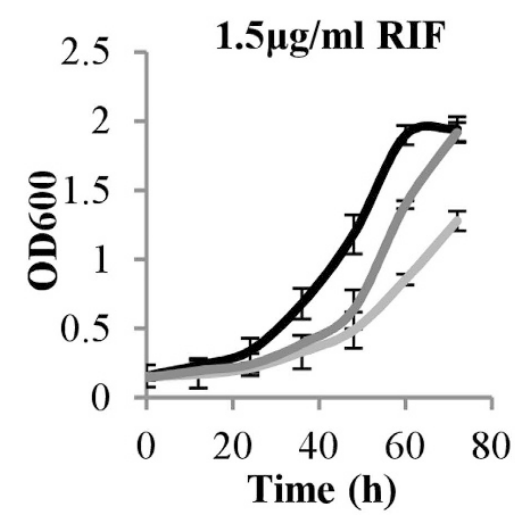

Figure 1. The effect of Ms4022 on mycobacterial growth in response to anti-tuberculosis drug rifampin (RIF). (A) Analysis of the structural characteristics of Ms4022. It contained a TetR_N superfamily domain within N domain. (B) Growth curves of the Ms/pMV261 and Ms/pMV261-Ms4022 were determined in the absence or presence of RIF. Ms/pMV261 and Ms/pMV261-Ms4022 were grown in 7H9 medium, which contains $30 \mu \mathrm{g} / \mathrm{ml}$ Kanamycin, without RIF (left panel) or $1.5 \mu \mathrm{g} / \mathrm{ml}$ RIF (right panel). (C) Ms/pMindD, $\Delta \mathrm{Ms} 4022 / \mathrm{pMindD}$ and $\Delta \mathrm{Ms} 4022 / \mathrm{pMindD}-\mathrm{Ms} 4022$ were grown in $7 \mathrm{H} 9$ medium, which contains $30 \mu \mathrm{g} / \mathrm{ml} \mathrm{Kanamycin}$, with no drug (left panel) or $1.5 \mu \mathrm{g} / \mathrm{ml} \mathrm{RIF} \mathrm{(right} \mathrm{panel).} \mathrm{For} \mathrm{all} \mathrm{these} \mathrm{assays,} \mathrm{aliquots} \mathrm{were} \mathrm{taken} \mathrm{at} \mathrm{the} \mathrm{indicated} \mathrm{times} \mathrm{and}$ the $\mathrm{OD}_{600}$ was measured. Each analysis was performed in triplicate, and standard errors were indicated as error bars.

immunoprecipitation in wild-type M. smegmatis but not in Ms4022-deleted M. smegmatis. The Ms4022 antiserum could not recover the negative control Ms4121p.

In conclusion, Ms4022 can bind with the 22 promoters of target genes both in vitro and in vivo.

Ms4022 positively regulates the expression of transport-related genes. The expressions of target genes were detected by quantitative real-time PCR (qRT-PCR) assays. As shown in Fig. 6B, the expression of seven transport-related genes was down-regulated in Ms/ $\Delta \mathrm{Ms} 4022$ compared with Ms/wt. As expected, the expression levels of these genes were up-regulated in the Ms/pMV261-Ms4022. The expression of Ms4121 without the conserved motif did not change considerably. Meanwhile, no signal was detected in the no-reverse transcriptase control. Several other transport-related genes were also activated by Ms4022 (Supplementary Fig. 6A 
A
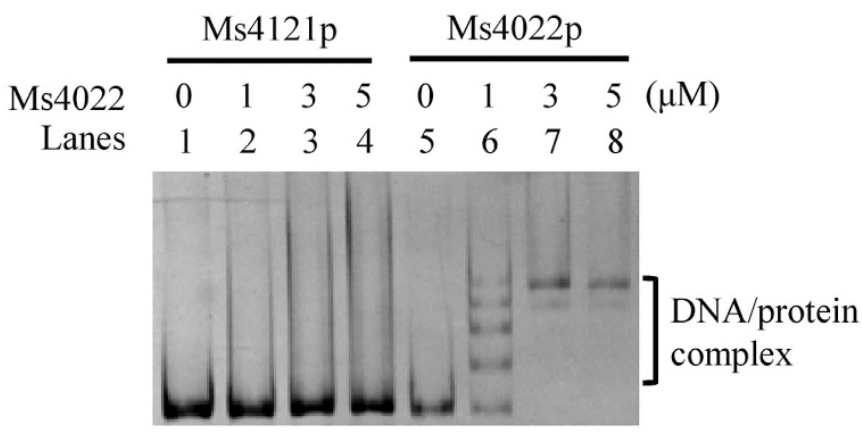

B

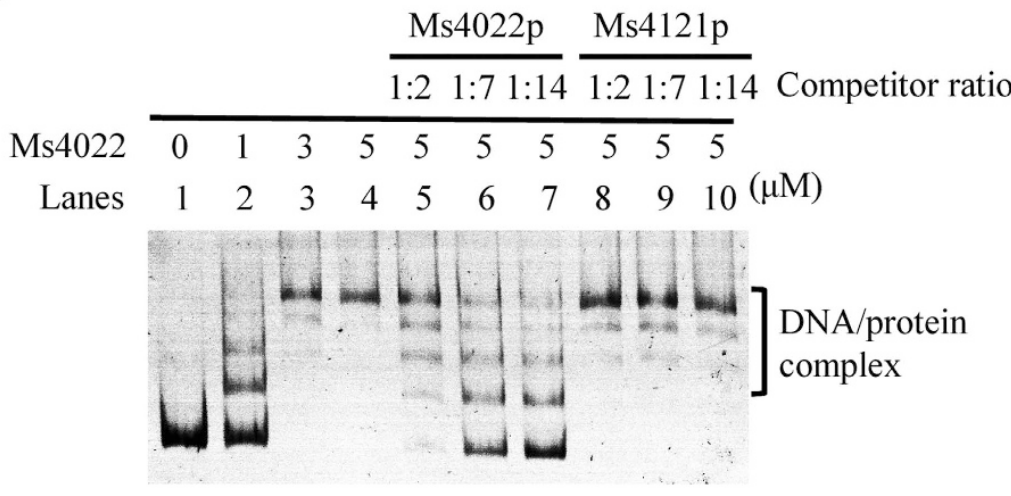

Figure 2. Specific binding of Ms4022 with its own promoter in vitro. (A) EMSA assays. Ms4022p or Ms4121p were co-incubated with various amount of Ms4022 protein as indicated. Ms4022 bound to Ms4022p, but not to Ms4121p, and form stable DNA/protein complexes. (B) EMAS assays for the specific binding of Ms4022 with Ms4022p. Unlabeled specific Ms4022 promoter and unspecific Ms4121 promoter were used to compete with the labeled Ms4022 promoter.

and B). These findings suggest that Ms4022 functions as a transcriptional activator and regulates the expression of transport-related genes.

Furthermore, the $\beta$-galactosidase experiment assay confirmed the regulation of Ms4022 to its target genes. Reporter plasmids were constructed and transformed into Ms/wt and Ms/ $\Delta$ Ms4022 (Fig. 6C). The expressions of lacZ connected with all four promoters (Ms1448p, Ms4886p, Ms5051p, and Ms6909p) were down-regulated in $\mathrm{Ms} / \Delta \mathrm{Ms} 4022$ compared with Ms/wt. However, the expressions of lacZ connected with the strong promoter $h s p 60$ had no difference in Ms/wt and Ms/ $\Delta \mathrm{Ms} 4022$. Similar results were obtained when the promoter was substituted by the negative control promoter Ms4121p. Again, lacZ connected with the null promoter exhibited no signal of galactosidase activity (Fig. 6C).

These results show that Ms4022 can function as a transcriptional activator and positively affect the expressions of transport-related genes.

Seven transport-related genes overexpressing M. smegmatisis strains showed significant RIF resistance. We transformed the overexpression plasmids of 14 transport-related genes in $M$. smegmatis and detected the growth and drug susceptibility of these recombinant strains. The growth curves of the recombinant strains showed that the Ms/pMV261-Ms1448, Ms/pMV261-Ms1613, and Ms/pMV261-Ms5278 strains grew much slower than the Ms/pMV261 strain in the 7H9 medium (Fig. 7A). The other recombinant strains did not affect the growth of M. smegmatis. The drug susceptibility of these strains was revealed by the growth curves in the presence of RIF. Seven transport-related genes overexpressing M. smegmatis strains (Ms/pMV261-Ms1613, Ms/ pMV261-Ms1448, Ms/pMV261-Ms2727, Ms/pMV261-Ms3310, Ms/pMV261-Ms5051, Ms/pMV261-Ms5278, and Ms/pMV261-Ms6909) grow faster than the wild-type control strain when RIF was present in the 7H9 medium (Fig. 7A,B).

These results indicate that the seven transport-related genes activated by Ms4022 could result in RIF resistance of M. smegmatis.

\section{Discussion}

A new TetR family transcriptional factor, Ms4022, was functionally characterized in M. smegmatis. We proved that the overexpression of Ms4022 caused RIF resistance in M. smgmatis through elevated levels of transport-related genes.

The genomes of both fast-growing M. smegmatis and pathogenic M. tuberculosis encode many putative drug transporters and membrane protein ${ }^{22}$. Several regulators and transporters involved in mycobacterial multidrug resistance have been demonstrated ${ }^{23}$. For example, $M$. tuberculosis iniBAC is involved in the tolerance to INH and $\mathrm{EMB}^{8}$. A histone-like protein Lsr2 negatively regulate the expression of iniBAC ${ }^{24}$. In addition, LfrA was the first efflux pump described in M. smegmatis, belonging to the major facilitator family (MFS) protein. LfrA 
A

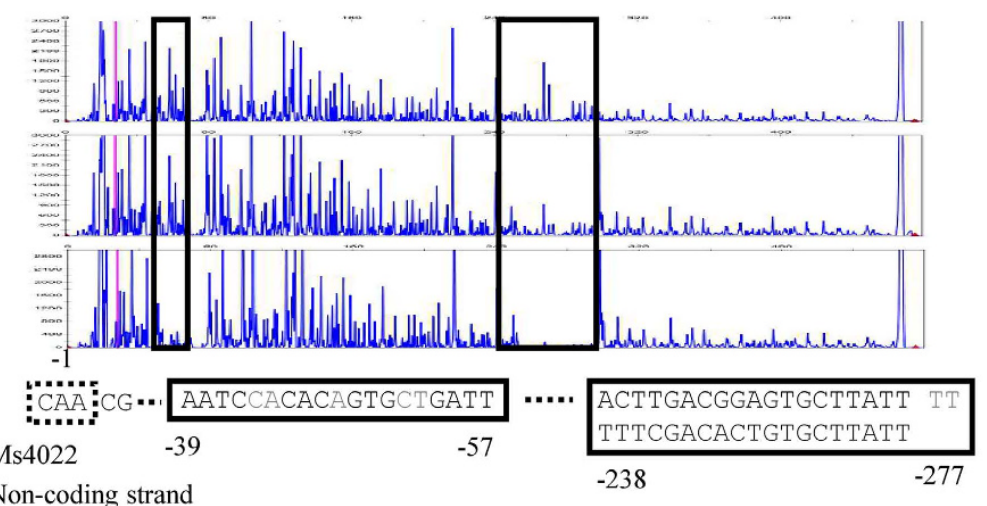

B

Motif 1 AATCAGCACTGTGTGGATT Motif 2 AATAAGCACTCCGTCAAGT

Motif 3 AATAAGCACAGTGTCGAAA

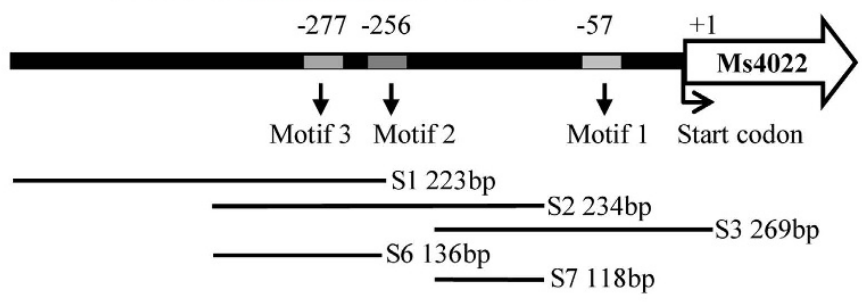

C

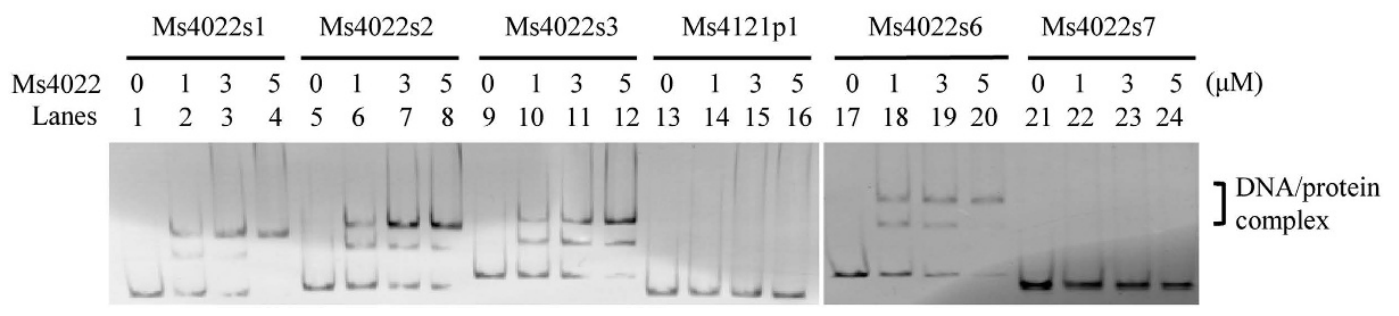

Figure 3. Identification of the DNA binding motif for Ms4022. (A) DNaseI footprinting assay. Ms4022p was incubated with increasing amounts of Ms4022 protein (from top to bottom), and digested by DNaseI. The protected region was shown with the box highlights. (B) Sequence and structural characteristics of the promoter region protected by Ms4022. The regions protected by Ms4022 were three $19 \mathrm{bp}$ sequences. Their approximate positions were also given in this diagram. The translation start codon of Ms4022 was indicated in curved arrow. DNA fragments that used in the following EMSA assays were showed as well. Those fragments may contain binding motifs of Ms4022. (C) EMSA assay for DNA fragments of different truncated. The promoter of Ms4022 was truncated to different length to detect the DNA-binding region of Ms4022 on its own promoter. Different DNA fragments s1, s2, s3, s6, s7 and Ms4121p1 were incubated with various amounts of Ms4022 protein. Ms4121p1 was used as the negative control.

overexpression contributes to the resistance of fluoroquinolones. LfrR, a TetR family transcriptional repressor, inhibits the expression of $\mathrm{LfrA}^{7}$. The deletion of $l f r R$ resulted in resistance to several drugs ${ }^{25}$. Usually, TetR family transcription factor binds a conserved palindromic sequence and represses the expression of target genes ${ }^{10}$. Unlike LfrR, Ms4022 acts as an activator and recognizes three19bp non-palindromic sequence motifs (motif 1 from -39 to -57 , motif 2 from -238 to -256 and motif 3 from -258 to -277 ) in its own promoter proved by EMSA and footprint assays. We got 315 potential target genes positively regulated by Ms4022. By analyzing the binding motif position of these genes, we found 95\% (133/140, motif 1), 96\% (130/136, motif 2$)$ and 92\% (68/74, motif 3) were outside the -35 region which is the RNA polymerase recognition site. The binding of Ms 4022 to promoters may located upstream of ribosomal binding site, and thus enhance the transcription of target genes ${ }^{26}$. For this case, transcriptional factor usually functions as an activator. For example, Ms6564 is a TetR family activator broadly affects the expressions of diverse genes in $M$. smegmatis ${ }^{15}$. Compared with the conserved region in target genes' motif, only $9 \mathrm{bp}$ shown high similarity. Therefore, the binding pattern of Ms4022 may be affected and regulated by its long C-terminal non-conserved region (Supplementary Fig. 5A). Ms4022 binds to those target promoters may favor the RNA polymerase to promote transcription of target genes.

In this study, we identified a TetR famlily regulator Ms4022 and seven transport related genes contributing drug resistance to RIF, but not to INH and EMB in M. smegmatis. They are Ms1448 (sulfate permease), Ms1613 (polar amino acid transport system permease), Ms2727 (glutamate transport system substrate-binding protein), 
A

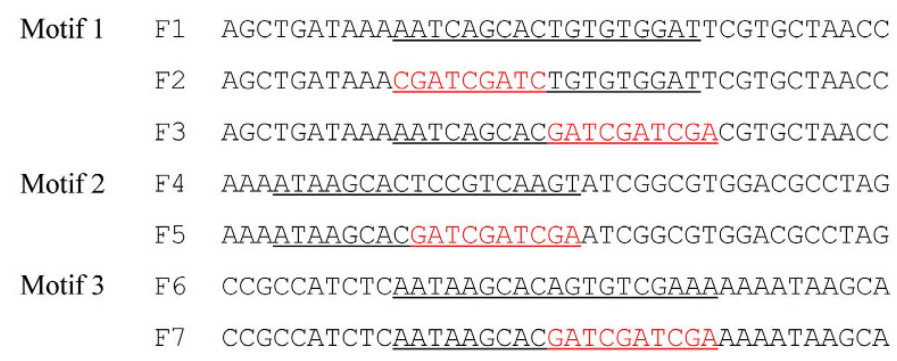

B

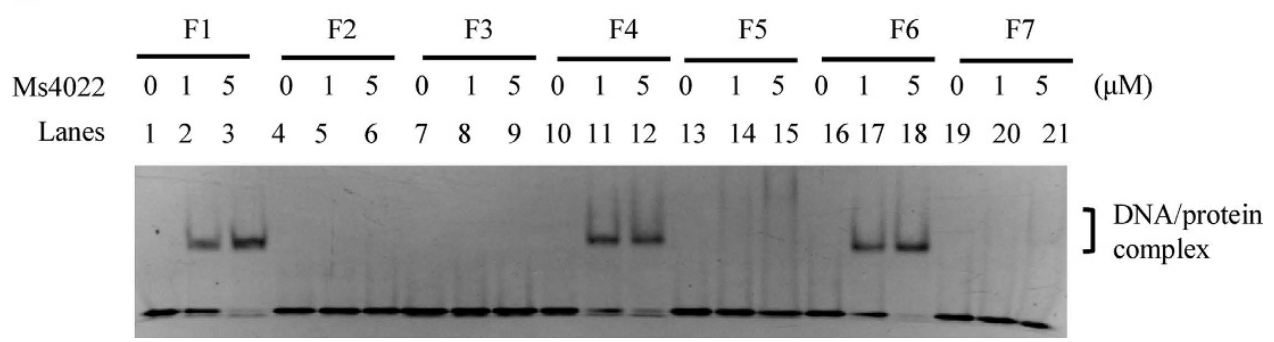

Figure 4. Specific binding of Ms4022 with the motifs. EMSA assays for the DNA-binding activity of Ms4022 on the DNA substrates with or without the complete binding motifs. Ms4022 could bind to F1, F4 and F6, but not F2, F3, F5 and F7 (with the mutant motif sequence).

A

The number of potential target genes

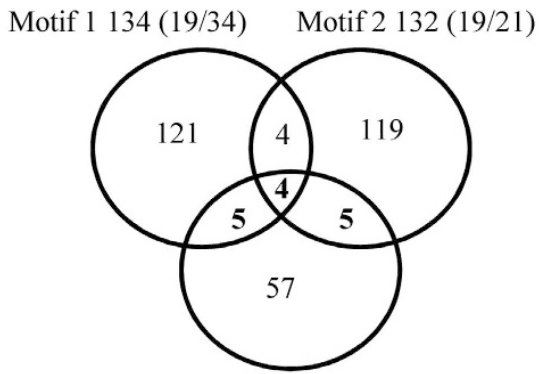

Motif $371(7 / 8)$

\section{B Functional categories of potential target genes}

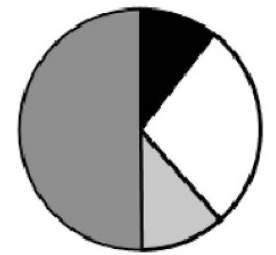

$\mathrm{ABC}$ transporter or membrane protein, $31,9.84 \%$

$\square$ hypothetical protein, $88,27.94 \%$

regulator protein, $36,11.43 \%$

Others, $160,50.79 \%$

Figure 5. The number and functional categories of Ms4022 potential target genes in M. smegmatis. (A) Motif 1(AATCAGCACTGTGTGGATT), Motif 2 (AATAAGCACTCCGTCAAGT) and Motif 3 (AATAAGCACAGTGTCGAAA) were used to search the promoters of M. smegmatis. The number of Ms4022 potential target genes in M. smegmatis were shown in Venn figure. Some promoters of the target genes were detected by EMSA assays (see also supplementary Fig. 4). In those randomly selected promoters, binding rates for promoters were given within the brackets. (B) A function classification of the target genes in the context of COG categories.

Ms3310 (integral membrane protein), Ms5051 (major facilitator superfamily protein), Ms5278 (transmembrane protein), and Ms6909 (amino acid ABC transporter permease). The possible mechanism would be a substrate preference to RIF. Transport related genes always show preference to a certain type of substrates. Based on the range of substrates, transporters can be divided into two categories. One is multi-substrates efflux system which shows broad substrate specificity. The other is single-substrate efflux system which tends to transport structural analogs. It is possible that 7 target genes of Ms4022 belong to single-drug efflux systems. Specific transport proteins have also been reported. For example, E. coli TetB can extrude tetracycline and a narrow range of close structural analogs ${ }^{27}$. In Bacillus cereus, BC4707 show a more specific role in the efflux of norfloxacin, and MmpL7 is a INH efflux in M. smegmatis ${ }^{28,29}$. Drug-specific response transcription factors also exist. Such as, FurA, OhrR and Ms0535 showed specific drug resistance ${ }^{20,30,31}$. In addition, Ms1448, Ms1613, and Ms5278 overexpressed strains could partially inhibit the growth of $M$. smegmatis. It appears that these target genes may be important in determining the RIF resistance of $M$. smegmatis only when their expression is increased. Interestingly, a homologous protein of Ms4022, MAV_1977, was also found in the pathogen M. avium. Six transport-related genes 
A

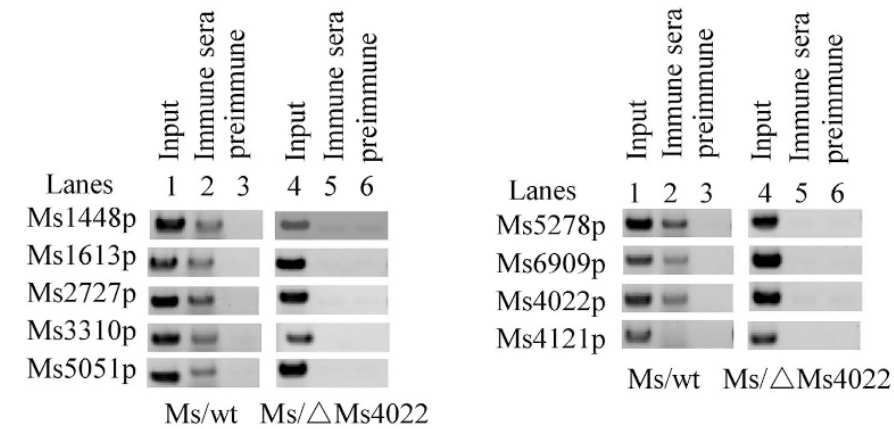

$\mathbf{B}$

口 Ms/wt

$\mathrm{Ms} / \triangle \mathrm{Ms4022}$

口 Ms/pMV261 Ms/pMV261-Ms4022

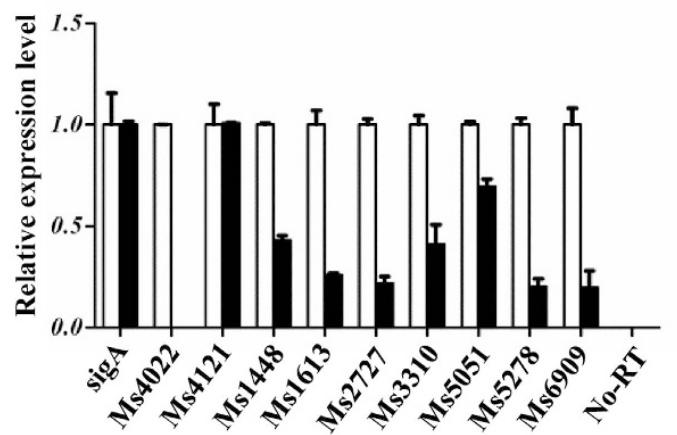

Target genes

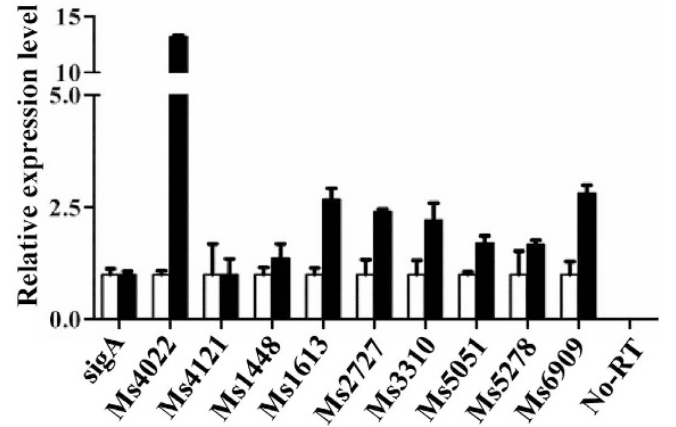

Target genes

C

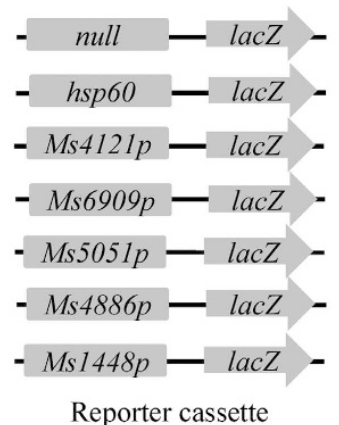

Reporter cassette

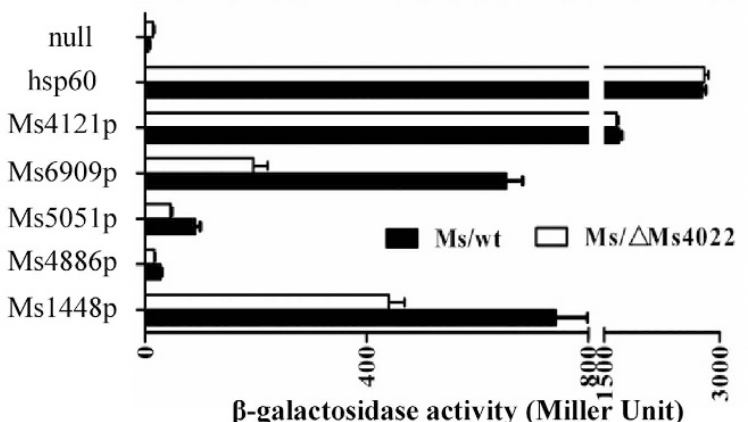

$\beta$-galactosidase activity (Millēr Unit)

Figure 6. Interaction of Ms4022 with the promoter of its target genes in vivo and expression assays of target genes in different recombinant strains. (A) ChIP assays for the binding of Ms 4022 to promoters of target genes. ChIP using pre-immune or immune sera rose against Ms4022. DNA recovered from the immunoprecipitates was amplified with primers specific for the target genes or an unrelated mycobacterial promoter of Ms4121. (B) Quantitative real-time PCR (qRT-PCR) assay for the regulation Ms4022 to its target genes in M.smegmatis. The relative expression levels of the genes were normalized using sigA rRNA gene as an invariant transcript, and an unrelated promoter gene Ms4121 was used as negative control. The cDNA of $\mathrm{Ms} /$ wt or Ms/pMV261was negative control. Data were analyzed using the $2^{-\Delta \Delta \mathrm{Ct}}$ method. The P-values of the relative expression data were calculated by unpaired two-tailed Student's T-test using GraphPad Prism5. (C) $\beta$-galactosidase activity assay. Left panel: Schematic representation of plasmids containing null-lac $Z$ and promoter-lacZ. All of the plasmids were transformed into the Ms/wt or Ms/ $\Delta \mathrm{Ms} 4022$.The activity of $\beta$ -galactosidase was further examined and presented as Miller units. Null-lacZ, hsp60-lacZ and Ms4121p-lacZ were used as controls. Relative expression data were analyzed for statistical significance, $\mathrm{P} \leq 0.05$.

(except Ms3310) have their homologs in pathogen M. avium and M. tuberculosis. Therefore, our findings in $M$. smegmatis provided important clues for further understanding of the function of transport-related genes and their regulation of bacterial drug resistance in pathogenic mycobacteria. Unexpectedly, Ms4022 has no homolog in M. tuberculosis. The regulation of target genes remains to be identified in M. tuberculosis.

In sum, we presume that Ms4022 is a novel TetR family transcription factor that contributes to the RIF resistance of M. smegmatis. The findings of this study enhance our understanding of transport-related gene regulation and drug resistance in mycobacteria. 
A

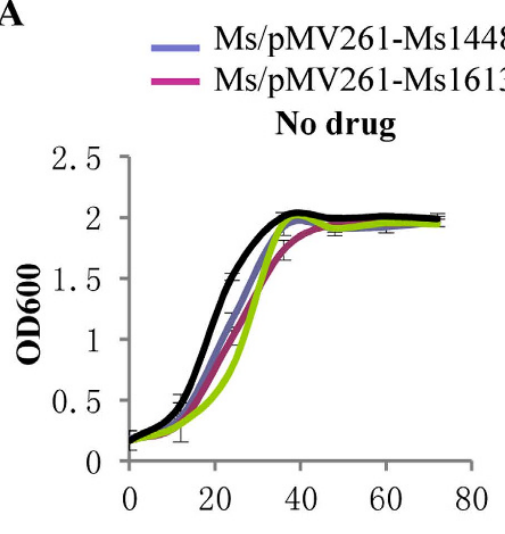

Time (h)

B

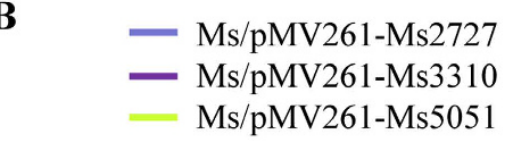

No drug

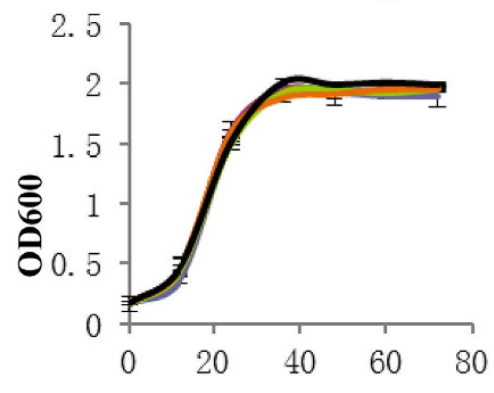

Time (h)
Ms/pMV261-Ms5278

- Ms/pMV261

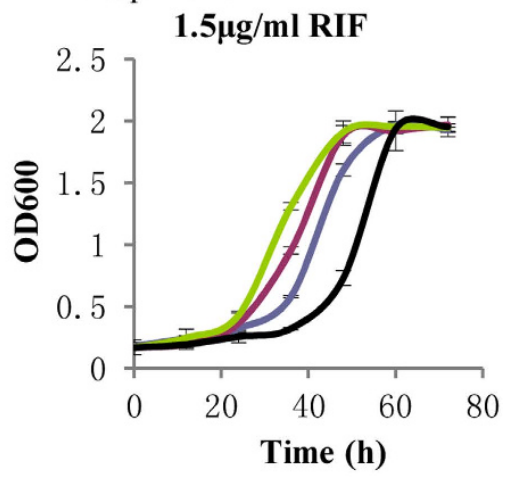

- Ms/pMV261-Ms6909
- Ms/pMV261

$1.5 \mu \mathrm{g} / \mathrm{ml}$ RIF

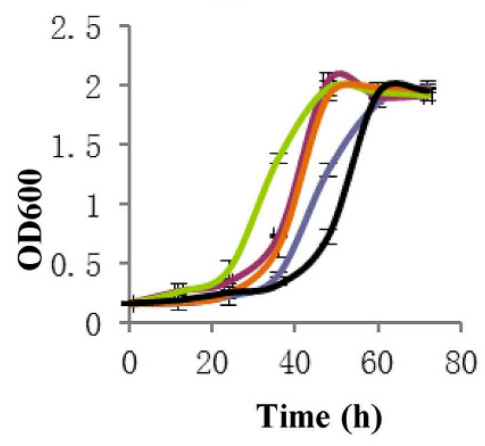

Figure 7. The growth curves of seven target genes overexpressed in M. smegmatis in response to RIF. All strains were grown in $7 \mathrm{H} 9$ medium that contains $30 \mu \mathrm{g} / \mathrm{ml}$ Kan without or with $1.5 \mu \mathrm{g} / \mathrm{ml} \mathrm{RIF.} \mathrm{Ms1448,} \mathrm{Ms1613}$ and Ms5278 overexpressing strains affected M. smegmatis growth. All of these seven target genes overexpressed strains showed resistance to RIF. Aliquots were taken at the indicated times and the OD600 was measured. Each analysis was performed in triplicate. (A) Growth curves of Ms1448, Ms1613 and Ms5278. (B) Growth curves of Ms2727, Ms3310, Ms5051 and Ms6909.

\section{References}

1. Alekshun, M. N. \& Levy, S. B. Molecular mechanisms of antibacterial multidrug resistance. Cell 128, 1037-1050 (2007).

2. Walsh, C. Molecular mechanisms that confer antibacterial drug resistance. Nature (London) 406, 775-781 (2000).

3. Sandhu, G. K. Tuberculosis: current situation, challenges and overview of its control programs in India. Journal of global infectious diseases 3, 143-150 (2011).

4. Lomovskaya, O. \& Watkins, W. J. Efflux pumps: Their role in antibacterial drug discovery. Curr Med Chem 8, 1699-1711 (2001).

5. Marquez, B. Bacterial efflux systems and efflux pumps inhibitors. Biochimie 87, 1137-1147 (2005).

6. Putman, M., van Veen, H. W. \& Konings, W. N. Molecular properties of bacterial multidrug transporters. Microbiology and molecular biology reviews: MMBR 64, 672-693 (2000).

7. Liu, J., Takiff, H. E. \& Nikaido, H. Active efflux of fluoroquinolones in Mycobacterium smegmatis mediated by LfrA, a multidrug efflux pump. J. Bacteriol. 178, 3791-3795 (1996).

8. Colangeli, R. et al. The Mycobacterium tuberculosis iniA gene is essential for activity of an efflux pump that confers drug tolerance to both isoniazid and ethambutol. Molecular Microbiology 55 (2005).

9. Hao, P. et al. The role of ABC efflux pump, Rv1456c-Rv1457c-Rv1458c, from Mycobacterium tuberculosis clinical isolates in China. Folia microbiologica 56, 549-553 (2011).

10. Ramos, J. L. et al. The TetR family of transcriptional repressors. Microbiology And Molecular Biology Reviews 69, 326-356 (2005).

11. Grkovic, S., Brown, M. H., Roberts, N. J., Paulsen, I. T. \& Skurray, R. A. QacR is a repressor protein that regulates expression of the Staphylococcus aureus multidrug efflux pump QacA. The Journal of biological chemistry 273, 18665-18673 (1998).

12. Lomovskaya, O., Lewis, K. \& Matin, A. EmrR is a negative regulator of the Escherichia coli multidrug resistance pump EmrAB. Journal of bacteriology 177, 2328-2334 (1995).

13. Engohang-Ndong, J. et al. EthR, a repressor of the TetR/CamR family implicated in ethionamide resistance in mycobacteria, octamerizes cooperatively on its operator. Molecular Microbiology 51, 175-188 (2004).

14. Li, W. \& He, Z.-G. LtmA, a novel cyclic di-GMP-responsive activator, broadly regulates the expression of lipid transport and metabolism genes in Mycobacterium smegmatis. Nucleic Acids Research 40, 11292-11307 (2012).

15. Yang, M., Gao, C., Cui, T., An, J. \& He, Z.-G. A TetR-like regulator broadly affects the expressions of diverse genes in Mycobacterium smegmatis. Nucleic Acids Research 40, 1009-1020 (2012). 
16. Rao, M., Liu, H., Yang, M., Zhao, C. \& He, Z.-G. A Copper-responsive Global Repressor Regulates Expression of Diverse Membraneassociated Transporters and Bacterial Drug Resistance in Mycobacteria. Journal Of Biological Chemistry 287, 39721-39731 (2012).

17. Livak, K. J. \& Schmittgen, T. D. Analysis of relative gene expression data using real-time quantitative PCR and the 2-DELTADELTACT method. Methods (Orlando) 25, 402-408 (2001).

18. Lun, S. et al. Indoleamides are active against drug-resistant Mycobacterium tuberculosis. Nature Communications 4 ,2907 (2013).

19. Gao, C. H., Yang, M. \& He, Z. G. An ArsR-like transcriptional factor recognizes a conserved sequence motif and positively regulates the expression of phoP in mycobacteria. Biochemical And Biophysical Research Communications 411, 726-731 (2011).

20. Hu, J., Zhao, L. \& Yang, M. A GntR family transcription factor positively regulates mycobacterial isoniazid resistance by controlling the expression of a putative permease. Bmc Microbiol 15, 214 (2015).

21. Crooks, G. E., Hon, G., Chandonia, J. M. \& Brenner, S. E. WebLogo: A sequence logo generator. Genome Research 14, 1188-1190 (2004).

22. Cole, S. T. et al. Deciphering the biology of Mycobacterium tuberculosis from the complete genome sequence. Nature 393, 537-544 (1998).

23. Lee, E. W. et al. Functional cloning and expression of emeA, and characterization of EmeA, a multidrug efflux pump from Enterococcus faecalis. Biol Pharm Bull 26, 266-270 (2003).

24. Colangeli, R. et al. Transcriptional regulation of multi-drug tolerance and antibiotic-induced responses by the histone-like protein Lsr2 in M-tuberculosis. PLoS Pathog. 3, 780-793 (2007).

25. Buroni, S. et al. LfrR is a repressor that regulates expression of the efflux pump LfrA in Mycobacterium smegmatis. Antimicrob. Agents Chemother. 50, 4044-4052 (2006).

26. Browning, D. F. \& Busby, S. J. W. The regulation of bacterial transcription initiation. Nat. Rev. Microbiol. 2, 57-65 (2004).

27. Levy, S. B. Active efflux mechanisms for antimicrobial resistance. Antimicrobial agents and chemotherapy 36, 695-703 (1992).

28. Simm, R. et al. BC4707 Is a Major Facilitator Superfamily Multidrug Resistance Transport Protein from Bacillus cereus Implicated in Fluoroquinolone Tolerance. PLoS One 7 (2012).

29. Pasca, M. R., Guglierame, P., De Rossi, E., Zara, F. \& Riccardi, G. mmpL7 gene of Mycobacterium tuberculosis is responsible for isoniazid efflux in Mycobacterium smegmatis. Antimicrob. Agents Chemother. 49, 4775-4777 (2005).

30. Saikolappan, S., Das, K. \& Dhandayuthapani, S. Inactivation of the Organic Hydroperoxide Stress Resistance Regulator OhrR Enhances Resistance to Oxidative Stress and Isoniazid in Mycobacterium smegmatis. J. Bacteriol. 197, 51-62 (2015).

31. Zahrt, T. C., Song, J., Siple, J. \& Deretic, V. Mycobacterial FurA is a negative regulator of catalase-peroxidase gene katG. Molecular Microbiology 39, 1174-1185 (2001).

\section{Acknowledgements}

This work was supported by the National Natural Science Foundation of China $(81471996,31301079$ and 31421064), the China Postdoctoral Science Foundation (2013M540587 and 2014T70709), and Changjiang Scholar Program of Chinese Ministry of Education (To Z-G He).

\section{Author Contributions}

Planned experiments: M.Y. and Z.G.H.; Performed experiments: H.L.; Analyzed data: H.L. and M.Y.; Contributed reagents or other essential material: Z.G.H.; Wrote the paper: M.Y., H.L. and Z.G.H.

\section{Additional Information}

Supplementary information accompanies this paper at http://www.nature.com/srep

Competing financial interests: The authors declare no competing financial interests.

How to cite this article: Liu, H. et al. Novel TetR family transcriptional factor regulates expression of multiple transport-related genes and affects rifampicin resistance in Mycobacterium smegmatis. Sci. Rep. 6, 27489; doi: 10.1038/srep27489 (2016).

(c) (i) This work is licensed under a Creative Commons Attribution 4.0 International License. The images or other third party material in this article are included in the article's Creative Commons license, unless indicated otherwise in the credit line; if the material is not included under the Creative Commons license, users will need to obtain permission from the license holder to reproduce the material. To view a copy of this license, visit http://creativecommons.org/licenses/by/4.0/ 